\title{
Mathematics, Physics, and Calabi-Yau Manifolds
}

\section{by Shing-Tung Yau ${ }^{\dagger}$ and Steve Nadis ${ }^{\ddagger}$}

I'd like to talk about how mathematics and physics can come together to the benefit of both fields, particularly in the case of Calabi-Yau spaces and string theory. This happens to be the subject of the new book I co-authored, The Shape of Inner Space, which tells the story of those spaces. It also tells some of my own story and a bit of the history of geometry as well.

In that spirit, I'm going to back up and talk about my personal introduction to geometry and how I ended up spending much of my career working at the interface between math and physics. Along the way, I hope to give people a sense of how mathematicians think and approach the world. I also want people to realize that mathematics does not have to be a wholly abstract discipline, disconnected from everyday phenomena, but is instead crucial to our understanding of the physical world.

\section{Riemannian Geometry}

When I arrived in Berkeley in 1969 for graduate study, I learned that the concept of geometry had gone through a radical change in the 19th century, thanks to the contributions of Gauss and Riemann. Riemann revolutionized our notions of space, freeing up mathematics in the process.

Objects no longer had to be confined to the flat, linear space of Euclidean geometry. Riemann instead proposed a much more abstract conception of space-of any possible dimension-in which we could describe distance and curvature. In fact, one can develop a form of calculus that is especially suited to such an abstract space.

About 50 years later, Einstein realized that this kind of geometry, which involved curved spaces, was exactly what he needed to unify Newtonian gravity with special relativity. This insight culminated in his famous theory of general relativity.

I learned about Riemannian geometry during my first year at Berkeley in 1969. It was different from the classical geometry that I studied in college in Hong Kong, where we focused on curves and surfaces in linear space. At Berkeley, I took courses on algebraic topology, Riemannian geometry, and partial differential equations. I

\footnotetext{
${ }^{\dagger}$ Department of Mathematics, Harvard University

${ }^{\ddagger}$ Steve Nadis is a contributing editor to Astronomy magazine.
}

also audited courses on many other subjects, including general relativity, taking in as much information as I could possibly assimilate.

I spent all my free time in the mathematics library, which served as my unofficial office, where I constantly searched for interesting articles to pass the time. During the Christmas holiday of that year, when everybody else went home, I read John Milnor's paper in the Journal of Differential Geometry on the relation of the fundamental group to the curvature of a manifold. Milnor referred to another paper by Preissman that sounded interesting to me.

From these papers, I learned that if the space has negative curvature, there is a strong constraint on the "fundamental group"-a concept from topology. Such a group consists of closed loops in that space, each of which has an initial point fixed. The elements of this group, which can be deformed to each other, are considered equivalent. Preissman's theorem says that in the fundamental group of manifolds with negative curvature, every two commuting elements can be written as a multiple of some other element in the group. This was intriguing, and I started to toy around with Preissman's paper, trying to see what would happen if the space is allowed to have non-positive curvature. This was the first time I got into statements linking the curvature of a space-a precise description of the geometry-to a much cruder, more general way of characterizing shape, which we call topology.

I wrote down my generalization of Preissman's theorem, which links topology to geometry. While I was photocopying those notes in the Xerox room, I ran into Arthur Fisher, a mathematical physicist. He insisted on knowing what I had written. After reading through my notes, he told me that any principle that related curvature with topology would be useful in physics. His comments have stayed with me ever since.

\section{General Relativity}

We learned through special relativity that space and time should not be treated separately but should instead be merged together to form space-time. Einstein struggled in his attempt to obtain a fundamental description of gravity. But he got some help from his friend Marcel 
Grossman, a mathematician, who told him of the work of other mathematicians, Riemann and Ricci.

Riemann provided the framework of abstract space, as well as the means for defining distance and curvature in such a space. Riemann thus supplied the background space or setting in which gravity, as Einstein formulated it, plays out.

But Einstein also drew on the work of Ricci, who defined a special kind of curvature that could be used to describe the distribution of matter in spacetime. In fact, the Ricci curvature can be viewed as the trace of the curvature tensor. A remarkable feature of this curvature is that it satisfied the conservation law due to the identity of Bianchi. And it was exactly this conservation law that enabled Einstein to provide a geometric picture of gravity. Rather than considering gravity as an attractive force between massive objects, it could instead be thought of as the consequence of the curvature of spacetime due to the presence of massive objects. The precise way in which spacetime is curved tells us how matter is distributed.

To those readers interested in history, it is always instructive to find out what Einstein, himself, had to say on the subject. "Since the gravitational field is determined by the configuration of masses and changes with it, the geometric structure of this space is also dependent on physical factors," he wrote. "Thus, according to this theory, space is-exactly as Riemann guessed-no longer absolute; its structure depends on physical influences. [Physical] geometry is no longer an isolated, self-contained science like the geometry of Euclid."

But it still took Einstein many years to formulate his famous field equations. First he developed the special theory of relativity, establishing the equivalence of so-called inertial frames of reference, which he presented in 1905. A couple of years later, he realized that gravity could not be treated within special relativity, which was a linear theory, but instead needed to be treated in a separate, nonlinear theory. He then began to work on the latter theory, which came to be known as general relativity, admitting that "it took me a long time to see what coordinates at all meant in physics." The notion of equivalence, which held that the laws of gravity should be true in any coordinate system, had been his guiding principle. By 1912 he started to realize that the gravitational potential should be described by a second-order symmetric tensor-a Riemannian metric with a Lorentzian signature.

Two additional problems had to be solved as well, which Einstein noted: 1) How can a field law, expressed in terms of the special theory of relativity, be transferred to the case of a Riemannian metric? 2) What are the laws that determine the Riemannian metric itself?

He worked on these problems from 1912 to 1914 with Grossman. Together they determined that the mathematical methods for solving the first problem could be found in the differential calculus of Ricci and Levi-Civita. They further discovered that the solution of the second problem depended on a mathematical construction ("differential invariants of the second order") that had already been established by Riemann.

However, his collaboration with Grossman did not lead to the final form of the field equation of gravity, as the equation they found was not covariant and did not satisfy the conservation law. In November 1915 he finally found the correct version of his equation, which was around the same time that David Hilbert did so independently. But Einstein carried things an important step further, as he alone was able to link his theory with "the facts of astronomical experience."

Reflecting on his accomplishment, Einstein wrote: "In the light of the knowledge attained, the happy achievement seems almost a matter of course, and any intelligent student can grasp it without too much trouble. But the years of anxious searching in the dark, with their intense longing, their alternations of confidence and exhaustion, and the final emergence into the light-only those who have experienced it can understand that."

Einstein's struggle to understand gravity is remarkable and his success in this area even more so. One thing that is resoundingly apparent is the critical contribution of Riemannian geometry to that effort.

When I looked at the equations of Einstein more than a half century later, I was intrigued by the fact that matter only controls part of the curvature of space-time. I wondered whether we could construct a space-time that is a vacuum, and thus has no matter, yet its curvature is still pronounced, meaning that its gravity would be nonzero. Well, the famous Schwarzschild solution to Einstein's equations is such an example. This solution applies to a non-spinning black hole-a vacuum that, curiously, has mass owing to its extreme gravity. But that solution admits a singular point, or singularity - a place where the laws of physics break down.

I became interested in a different situation-a smooth space, without a singularity, that was compact and closed, unlike the open, extended space of the Schwarzschild solution. The question was: Could there be a compact space that contained no matter-a closed vacuum universe, in other words-whose force of gravity was nontrivial? I was obsessed with this question and believed that such a space could not exist. If I could prove that, I was sure that it would be an elegant theorem in geometry.

\section{Calabi Conjecture}

When I started thinking about this in the early 1970s, I did not realize that the geometer Eugenio Calabi had posed almost the exact same question. Calabi framed the problem in fairly complicated mathematical language-involving difficult concepts like Kähler manifolds, Ricci curvature, and Chern classes-that ostensibly had nothing to do with physics. Yet his abstract conjecture could also be framed in terms of Einstein's theory of general relativity. The additional information that he put in is that the space should admit some kind of internal 
symmetry called supersymmetry - a term coined by physicists. (Expressed in the language of geometry, this means an internal symmetry created by some constant spinors-constant in this case meaning spinors that are parallel. In the case of six-dimensional space, spaces with nontrivial constant spinors are Kähler manifolds unless the space is the Cartesian product of lower-dimensional spaces.) In that context, Einstein's question translated to: Can there be gravity, or the curving of space, in a closed vacuum-a compact supersymmetric space that has no matter?

For about three years, my friends and I tried to prove that the class of spaces proposed by Calabi could not exist. We, along with many others, considered them to be "too good to be true." We were skeptical not only because the conjecture argued for the existence of a closed vacuum with gravity but also because it implied that there was a systematic way of constructing many such examples. Despite the reasons we had for finding Calabi's argument dubious, try as we might, we could not prove that such spaces do not exist.

In the spring of 1973, I was an assistant professor at Stony Brook. I had some correspondence with Robert Osserman on surface theory, and he seemed interested in my work on minimal surfaces. Since my girlfriend was in California at the time, I decided to ask whether I might be able to come to Stanford during the next year. To my surprise, Osserman replied immediately and offered me a visiting position.

In late May of that year, I drove across the country with a graduate student. It was a long journey, and quite an experience, as both of us were relatively new to driving. Fortunately, I made it to Berkeley intact, with both the vehicle and my driving companion in one piece. There I met up with my friend S.-Y. Cheng and together we went to Stanford to settle down. I worked hard on some papers that were to be presented at a huge three-week conference at Stanford in August.

Osserman and my teacher S.S. Chern organized the conference. Perhaps my connections with them allowed me to present not one but two talks at this conference. But when I told some friends, while the meeting was underway, that I'd just found a counterexample to the Calabi conjecture, many geometers insisted that I give a separate presentation that evening. About 30 geometers gathered together on the third floor of the math building. The audience included Calabi, Chern, and other prominent mathematicians. I described my construction, and everybody seemed happy with it.

Calabi himself advanced an argument as to why this approach should work. At the end of the conference, Chern announced that this counterexample was, arguably, the best outcome of the entire conference. I was astonished but happy.

However, about two months later, reality set in. Calabi wrote me a letter regarding some points in my argument that he could not understand. When I received his letter, I immediately realized that I had made a mistake. I tried hard to come up with a new argument, working for two weeks straight with practically no sleep, pushing myself to the brink of collapse. Each time I found a possible counterexample, I soon found a subtle reason as to why it could not work. After many such abortive attempts, I concluded that the conjecture must be correct after all. Once I made up my mind, I switched gears completely, putting all my energies into proving it right. I finally did so, several years later, in 1976. An additional bonus was that many of my failed counterexamples became important theorems of their own years later when I finally proved that the conjecture was correct.

I should say that at the same Stanford conference, the physicist Robert Geroch gave a talk on an important question in general relativity called the positive mass conjecture, which holds that the total mass or energy in any closed physical system must be positive. Richard Schoen and I eventually proved this conjecture after some difficult calculations involving minimal surfaces and a lot of hard work. I still remember that the first hint of a possible solution hit us during a conversation we had while walking towards my apartment on the lawns of the west campus of Berkeley.

The experience led us to think more about general relativity, and we eventually proved some theorems about black holes. My work on the positive mass conjecture has led me to explore ideas about mass, in general, and the definition of "local" mass, in particular-notions that are, surprisingly, still poorly defined in general relativity, despite the theory's success for nearly a century.

My favorable interactions with general relativists also made me more open to collaborating with physicists in the development of string theory, although that didn't come until several years later.

In my proof of the Calabi conjecture, I found a general mechanism to construct spaces satisfying Calabi's equations, which are now called Calabi-Yau spaces. I had a strong sense that I had somehow stumbled onto a beautiful piece of mathematics. And as such, I felt it must be relevant to physics and to our deepest understanding of nature. However, I did not know exactly where these ideas might fit in, as I didn't know much physics at the time.

\section{String Theory}

In 1984 I received phones calls from two physicists, Gary Horowitz and Andy Strominger. They were excited about a model for describing the vacuum state of the universe, based on a new theory called string theory.

String theory is built on the assumption that particles, at their most basic level, are made of vibrating bits of strings - and exceedingly tiny strings at that. In order for the theory to be consistent with quantum mechanics (at least in some versions of string theory), spacetime requires a certain symmetry built into it called supersymmetry. Spacetime is also assumed to be ten-dimensional. 
Horowitz and Strominger became interested in the multidimensional spaces whose existence I proved, mathematically, in my confirmation of the Calabi conjecture. They believed that these spaces might play an important role in string theory, as they seemed to be endowed with the right kind of supersymmetry-a property deemed essential to the theories they were working on. They asked me if their assessment of the situation was correct and, to their delight, I told them that it was. Or at least might be.

Then I got a phone call from Edward Witten whom I'd met in Princeton the year before. Witten believed that this was the one of the most exciting eras in theoretical physics, just like the time when quantum mechanics was being developed. He told me that everyone who made contributions to quantum mechanics in early days left their mark on the history of physics. He said that the important discoveries of early string theorists, such as Michael Green and John Schwarz, could lead to the grand unification of all forces-the goal that Einstein had spent the last 30 years of his life working toward, although he did not succeed in the end.

Witten was now collaborating with Philip Candelas, Horowitz, and Strominger, trying to figure out the shape, or geometry, of the six "extra" dimensions of string theory. The physicists proposed that these six dimensions were curled up into a miniscule space, which they called Calabi-Yau space-part of the same family of spaces, which Calabi originally proposed and I later proved to exist.

String theory, again, assumes that spacetime has 10 dimensions overall. The three large spatial dimensions that we're familiar with, plus time, make up the four-dimensional spacetime of Einstein's theory. But there are also six additional dimensions hidden away in Calabi-Yau space, and this invisible space exists at every point in "real space," according to string theory, even though we can't see it.

The existence of this extra-dimensional space is fantastic on its own, but string theory goes much farther. It says that the exact shape, or geometry, of Calabi-Yau space dictates the properties of our universe and the kind of physics we see. The shape of Calabi-Yau space-or the "shape of inner space," as we put it in our bookdetermines the kinds of particles that exist, their masses, the ways in which they interact, and maybe even the constants of nature.

In their attempts to derive the particles of nature, theoretical physicists rely on something called the Dirac operator. Analyzing the spectrum of this operator reveals the variety of particles that we might see. Based on the principle of separation of variables on this ten-dimensional spacetime, which is the product of the four-dimensional spacetime with the six-dimensional Calabi-Yau space, we know that part of the spectrum is contributed by the Calabi-Yau space. Particles with nonzero spectrum will be extremely large if the diameter of the Calabi-Yau space is very small. We do not expect to observe any of these particles, as they would only appear at incredibly high energies.

But particles with zero spectrum are potentially observable and can be calculated from the topology of the Calabi-Yau space. This gives you an idea of why the topology of this tiny, six-dimensional space could play an important role in physics.

While Einstein had said the phenomenon of gravity is really a manifestation of geometry, string theorists boldly proclaimed that the physics of our universe is a consequence of the geometry of Calabi-Yau space. That's why string theorists were so anxious to figure out the precise shape of this six-dimensional space-a problem we're still working on today.

Witten was eager to learn more about Calabi-Yau spaces. He flew from Princeton to San Diego to talk with me about how to construct them. He also wanted to know how many Calabi-Yau spaces there were for physicists to choose among. Initially, physicists thought there might only be a few examples-a few basic topologies-which made the goal of determining the "internal" shape of our universe seem a lot more manageable. But we soon realized there were many more examples of Calabi-Yau spaces-many more possible topologies-than were originally anticipated. In the early 1980s, I guessed that there were tens of thousands of these spaces, and that number has grown considerably since then.

The task of figuring out the shape of inner space suddenly seemed more daunting, and perhaps even hopeless if the number of possibilities turned out to be infinite. The latter question has yet to be settled, although I have always thought that the number of Calabi-Yau spaces of any dimension is finite. That number is certain to be big, but I believe it is bounded. One reason for thinking that stems from a theorem by Kollár, Miyaoka, and Mori, which showed that for each dimension the number of compact manifolds (or spaces) with positive Ricci curvature is indeed finite. Calabi-Yau spaces are compact as well-meaning they cannot extend to infinity-but they have zero Ricci curvature, rather than positive Ricci curvature, so they should be considered a "borderline" case. Normally when something is proven true for spaces of positive curvature it is likely to be true for spaces of nonnegative curvature, which would thus include Calabi-Yau spaces. Moreover, after two-and-ahalf decades of investigating these spaces, we've found no hint of any method that would enable us to construct an infinite number of them.

The excitement over Calabi-Yau spaces started in 1984, when physicists first began to see how these complex geometries might fit into their new theories. That enthusiasm kept up for a few years, before waning. But interest in Calabi-Yau spaces picked up again in the late 1980s, when Brian Greene, Ronen Plesser, Philip Candelas, and others began exploring the notion of "mirror symmetry." 
The basic idea here was that two different Calabi-Yau spaces, which had different topologies and seemed to have nothing in common, nevertheless gave rise to the same physics. This established a previously unknown kinship between so-called mirror pairs of Calabi-Yau spaces.

A conjecture proposed in 1995 by Strominger, Yau, and Zaslow offered insights into the substructure of a Calabi-Yau space. According to the so-called SYZ conjecture, a six-dimensional Calabi-Yau space can essentially be divided into two, three-dimensional spaces. One of these spaces is a three-dimensional torus. First you take the torus and "invert" it, through an operation similar to switching its radius from $r$ to $1 / r$. When you combine the inverted torus with the other three-dimensional space, you'll have the mirror manifold of the original Calabi-Yau space. This conjecture provides a geometrical picture of mirror symmetry though it has only been proven for special cases and has not yet been proven in a general sense.

The connection between mirror manifolds, which was uncovered through physics, proved to be extremely powerful in the hands of mathematicians. When they were stumped trying to solve a problem involving one Calabi-Yau space, they could try solving the same problem on its mirror pair. On many occasions, this approach was successful. As a result, mathematical problems of counting curves that had defied resolution, sometimes for as long as a century, were now being solved. (The German mathematician Hermann Schubert investigated many of these problems in the 19th century.) And a branch of mathematics called enumerative geometry was suddenly rejuvenated. These advances gave mathematicians greater respect for physicists, as well as greater respect for string theory itself.

Mirror symmetry is an important example of what we call a duality. It sheds light on the deep geometry of Calabi-Yau space. It has also helped us solve some very difficult questions of counting rational curves of various degrees on the quintic with five variables, which is a kind of Calabi-Yau space.

This problem, named after Schubert, dates back to the 19th century. Schubert showed that the number of degree one rational curves on a quintic is 2,875 . In 1986, Sheldon Katz found that there are 609,250 degree two curves. Then around 1989, two Norwegian mathematicians Geir Ellingsrud and Stein Strømme found that number of degree three curves-based on algebraic geometry techniques-was 2,683,549,425. Relying on a string theory approach, a group of physicists, led by Candelas, arrived at a different number, $317,206,375$. The physicists, however, had used a formula that, up to then, had not been motivated by mathematical principles. As such, rigorous justification of that formula still awaited confirmation by mathematicians.

In January of 1990, I organized the first major meeting between string theorists and mathematicians at the urging of Isadore Singer. The event took place at the Mathematical Sciences Research Institute MSRI) in Berkeley. At this meeting there was a somewhat tense debate regarding who was right, Ellingsrud and Strømme or the Candelas team. The discrepancy between the two camps lasted a few months until the mathematicians discovered a mistake in their computer code. After they corrected that error, their number agreed perfectly with that put forth by the physicists. And ever since then, mathematicians have begun to appreciate the depth of the insight provided by the string theorists.

The episode also provided firm evidence that mirror symmetry had a mathematical basis. It took several years but by the mid-to-late 1990s a rigorous mathematics proof of mirror symmetry-and a validation of the Candelas, et al. formula - was finally achieved independently by Givental and Lian-Liu-Yau.

\section{Conclusion}

Before we get too carried away, we should bear in mind that string theory, as the name suggests, is just a theory. It has not been confirmed by physical experiments, nor have any experiments yet been designed that could put that theory to a definitive test. So the jury is still out on the question of whether string theory actually describes nature, which was, of course, the original intent.

On the positive side of the ledger, some extremely intriguing, as well as powerful, mathematics has been inspired by string theory. Mathematical formulae developed through this connection have proved to be correct, and will always remain so, regardless of the scientific validity of string theory. Although it is empirically unproven, string theory now stands as the only consistent theory that unifies the different forces. And it is beautiful. Moreover, the effort to unify the different forces of nature has unexpectedly led to the unification of different areas mathematics that at one time seemed unrelated.

We still don't know what the final word will be. In the past two thousand years, the concept of geometry has evolved over several important stages to the current state of modern geometry. Each time geometry has been transformed in a major way, the new version has incorporated our improved understanding of nature arrived at through advances in theoretical physics. It seems likely that we shall witness another major development in the 21 st century, the advent of quantum geometry-a geometry that can incorporate quantum physics in the small and general relativity in the large.

The fact that abstract mathematics can reveal so much about nature is something I find both mysterious and fascinating. This is one of the ideas that my coauthor and I have tried to get across in our book, The Shape of Inner Space. One of the objectives of this book is to explain how mathematicians view the world. At heart we're just scientists who look at nature from a different, more abstract point of view than the empiricists. But the work 
mathematicians do is still based on the truth and beauty of nature, the same as it is in physics. One idea I've tried to get across is the thrill of working at the interface between mathematics and physics, showing how important ideas flow through different disciplines, with the result being the birth of new and important subjects.

In the case of string theory, geometry and physics have come together to produce some beautiful mathematics, as well as some very intriguing physics. The mathematics is so beautiful, in fact, and it has branched out into so many different areas, that it makes you wonder whether the physicists might be onto something after all.

The story is still unfolding, to be sure. I consider myself lucky to have been part of it and hope to stay involved in this effort for as long as I can contribute. 